\title{
APERÇU SUR LES APPORTS RÉCENTS DANS LE DOMAINE DE LA BRYOLOGIE MÉDITERRANÉENNE (TAXONOMIE, CHOROLOGIE, SOCIOLOGIE ET ÉCOLOGIE)
}

\author{
Jean Pierre HÉBRARD
}

RÉSUMÉ: Durant la période 1979-1988, les travaux effectués dans le domaine de la taxonomie, de la chorologie et de la sociologie des bryophytes ont été très nombreux en Péninsule Ibérique, et à un degré moindre en France, en Corse, en Italie, en Sardaigne, en Sicile et en Grèce.

Au Proche-Orient, les publications récentes traitent essentiellement de bryofloristique et concernent surtout l'Egypte, Israël, la Jordanie et plus accessoirement le Liban. Par contre, en Afrique du Nord, l'exploration bryologique détaillée des pays du Maghreb reste à entreprendre.

Mots clés: Taxonomie, chorologie, sociologie, écologie, bryologie méditerranéenne.

RESUMEN: Durante el período 1979-1988 los trabajos efectuados en el campo de la taxonomía, de la corología y de la sociología de los Briófitos han sido muy numerosos en la Península Ibérica y, en menor grado, en Francia, Córcega, Italia, Cerdeña, Sicilia y Grecia.

En el Oriente Próximo, las publicaciones recientes tratan esencialmente de brioflorística y conciernen, sobre todo, a Egipto, Israel, Jordania y más accesoriamente a Líbano. Por el contrario, en Africa del Norte, la exploración briológica detallada de los países del Magreb queda por emprender.

Palabras clave: Taxonomía, corología, sociología, ecología, briología mediterránea.

\section{INTRODUCTION}

Du point de vue climatique, les régions méditerranéennes sont caractérisées par une température moyenne annuelle de 15 à $20^{\circ} \mathrm{C}$, par une période de sècheresse estivale, et en conséquence, par une répartition très particulière des précipitations au cours de l'année. Ce type de climat se rencontre, non seulement sur le pourtour de la mésogée, mais également en Amérique du Nord (Californie), au Chili, en Australie et en Afrique du Sud (Ozenda 1982).

Au cours de cet exposé, je tenterai de fournir un aperçu des données récentes (pour la période allant du début de l'année 1979 à la fin de 1988) concernant la 
taxonomie, la floristique, la chorologie, la sociologie et l'écologie des bryophytes de l'ensemble du bassin méditerranéen (Europe, Afrique du Nord et Proche-Orient).

Compte tenu de l'ampleur du sujet, mais également pour des raisons d'ordre floristique, seuls seront considérés ici les étages thermo- méso-et supraméditerranéens au sens d'Ozenda (1975). En effet, dans le nord du bassin, la bryoflore perd peu à peu son caractère méditerranéen lorsqu'on s'élève en altitude. Dès la frange supérieure de l'étage montagnard, les éléments circumboréal et arctico-alpin prédominent dans les chaînes alpine et pyrénéenne. Dans le sud du bassin, et notamment sur les montagnes d'Afrique du Nord, les muscinées thermophiles s'élèvent au contraire à des altitudes bien supérieures. Toutefois, les données relatives à ces régions demeurent encore extrêmement fragmentaires.

Enfịn, l'Albanie, la Turquie, Chypre et la Syrie seront rarement mentionnées, car je n'ai pas réusi à réunir pour ces états, une documentation récente suffisante.

\section{TAXONOMIE ET FLORISTIQUE}

A - La partie européenne du bassin méditerranéen et la Turquie

Une abondante documentation récente est disponible pour cet ensemble. Des listes critiques de taxons incluant les synonymes couramment utilisés ont été établies à petite ou à grande échelle.

A petite échelle, les check-lists de Corley et al. (1982: mousses) et de Grolle (1983: hépatiques) couvrent la totalité du continent européen (limites de Flora Europaea). Il s'agit dans les deux cas d'une énumération de taxons, dont la présence dans chaque pays n'est toutefois pas mentionnée.

Les contributions de Düll (1983: hépatiques, 1984 et 1985: mousses) adoptent les mêmes limites, avec en plus Chypre (As 5), les Canaries et Madère (Afr 5). Ici, les taxons infraspécifiques sont pris en considérations et la présence de chaque unité systématique dans chaque état est précisée.

A plus grande échelle, des check-lists sont actuellement disponibles seulement pour l'Espagne (Casas Sicart 1981: mousses, comportant la révision des espèces de détermination douteuse des herbiers espagnols), la Grèce et la Crète (Preston 1981 et 1984b: hépatiques, 1984a: mousses), la Sardaigne (Cortini Pedrotti 1983: mousses), la Sicile (Dia, Miceli et Not 1985: hépatiques, Dia, Miceli et Raimondo 1987: mousses) et la Turquie (Çetin 1988a: hépatiques, 1988b: mousses).

Ces importantes synthèses, et notamment celles de Düll et de Preston ont intégré les acquisitions de la taxonomie moderne, ainsi que les découvertes récentes effectuées lors de l'exploration bryofloristique de nombreux territoires d'Europe méridionale, en particulier dans l'extrême sud-ẹst du continent.

Toutefois, un certain nombre d'apports postérieurs à ces publications méritent d'être signalés. Parmi les espèces nouvelles pour la science, on peut citer Acaulon 
casasianum Brugues et Crum et Acaulon dertosense Casas, Sergio, Cros et Brugues, décrits d'Espagne, ainsi que Cinclidotus nyholmiae Çetin, décrit de Turquie.

D'autres bryophytes sont nouvelles, soit pour l'Europe [(entre autres: Bryoerythrophyllum inaequalifolium (Tayl)Zander: Hs ${ }^{(1)}$, Crossidiumaberrans Holz. et Bartr.: Hs, Ga, Fossombronia crispa Nees et F. zeyheri Steph.: Lu, Riella cossoniana Trab.: Hs)], soit pour certains pays [(Barbula ehrenbergii (Lor.) Fleisch.: Sa, Brachythecium mildeanum (Schimp.) Schimp. ex Milde: Lu, Bryum dunense A.J.E. Sm. et H.L.K. Whiteh.: Ga, Si, Bryum gemmilucens Wilcz. et Demar.: Gr, Cinclidotus aquaticus (Hedw.) B. et S.: Lu, Ephemerum sessile (Bruch) C. Müll.: Gr, Ephemerum stellatum Philib.: Hs, Lu, Eurhynchium speciosum (Brid.) Jur.: Sa, Eurhynchium striatum (Hedw.) Schimp.: Co, Fissidens algarvicus Solms: Co, Frullania muscicola Steph. et Frullania oakesiana Aust.: Lu, Grimmia pitardii Corb.: Lu, Grimmia tergestina Tomm. ex B., S. et G.: Gr, Gymnostomum luisieri (Sergio) Sergio ex Crundw, Co. Leptobarbula berica (De Not.) Lindb.: Gr, Lu, Mnium hornum Hedw.: Sa, Orthotrichum tenellum Bruch ex Brid.: Si, Pohlia wahlenbergii (Web. et Mohr) Andr.: Sa, Pottia bryoides (Dicks.) Mitt.: Sa, Pottia recta (With.) Mitt.: Lu, Pyramidula algeriensis Chudeau et Douin: Ga, Rhodobryum ontariense (Kindb.) Kindb.: Hs, Rhynchostegiella letourneuxii (Besch.) Broth.: Co, Riccia subbifurca Warnst. ex Crozals: Lu, Sematophyllum substrumulosum (Hampe) Britt.: Si, Tortula caninervis (Mitt.) Broth.: Ga, Weissia levieri (Limpr.) Kindb.: Lu, Weissia longifolia Mitt.: Gr, var angustifolia (Baumg.) Crundw. et Nyh.: Lu.]

Enfin, Ochyra, Sergio et Schumacker (1988) viennet de démontrer que Racomitrium levieri Kindb. et $R$. dixonii A. Mach. du Portugal, sont en réalité identiques à Racomitrium lamprocarpum (C. Müll.) Jaeg. de l'hémisphère austral.

Beaucoup de ces apports proviennent de la Péninsule Ibérique, dont l'exploration bryofloristique se poursuit activement, notamment dans le sud de l'Espagne (citons entre autres Ros et Guerra 1987a: bryophytes terricoles de la région de Murcia) et au Portugal (bryoflore des formations calcaires, cf. Sergio et Sim-Sim 1985, Sergio et al. 1984, 1987, 1988). La quantité de travaux publiés pour la Péninsule Ibérique est si considérable, qu'il n'est pas possible de les énumérer ici. Le lecteur pourra consulter notamment la bibliographie très complète de Casas, Brugues et Cros (1984).

En France méridionale, les recherches ont surtout concerné le sud-est. Il s'agit en particulier des reliefs calcaires d'altitude peu élevée dans les départements des Bouches-du-Rhône et du Var (Hébrard 1987a: 85 mousses et 6 hépatiques du piémont de la Sainte-Victoire; Werner 1989), plus rarement dans les Alpes Maritimes (Werner et Hébrard 1986: 35 mousses et 2 hépatiques de la basse vallée du Var).

Ailleurs, des données récentes sont disponibles pour les grands causses de l'Aveyron, de la Lozère et du Gard (Pierrot, Rogeon et Houmeau 1983: Larzac, Causses Noir, Méjean et de Séverac, 161 mousses et 43 hépatiques). Elles manquent par contre totalement pour les départements de l'Aude, du Gard et de l'Hérault, et pour les parties basses des Pyrénées Orientales.

En Corse, outre une liste de 68 mousses et 15 hépatiques méconnues, rares ou 
nouvelles pour l'île (Hébrard 1986), l'inventaire bryologique détaillé d'un petit territoire (réserve naturelle de Scandola) a été publié (Hébrard 1988: 42 mousses et 15 hépatiques).

En ce qui concerne le territoire italien (Sicile et Sardaigne incluses), Cortini Pedrotti et Tosco (1988) viennent de fournir un aperçu des recherches bryologiques effectuées par les auteurs italiens, entre 1888 et 1988.

Ce travail, bien documenté, résume l'essentiel de l'activité des bryologues dans différents domaines (systématique et taxonomie, flores, catalogues, généraux et régionaux, chorologie, bryogéographie, phytocénotique et écologie), et fait le point sur l'état d'avancement de l'exploration bryofloristique dans les provinces. Il ressort de cette contribution, et de la «bibliografia briologica d'Italia» (Cortini Pedrotti 1986), qu'entre 1979 et 1984, l'exploration bryofloristique des parties méditerranéennes, a surtout été importante en Sicile et dans l'archipel toscan.

Ultérieurement, alors que Cortini Pedrotti (1985) a dressé une liste des bryophytes des environs du lac Trasimène (Ombrie), Andreani et Cortini Pedrotti (1986) ont étudié une réserve naturelle (forêt mix te de Quercus cerris L. et de Quercus pubescens Willd.), près de Macerata (Marche). La bryoflore, caractéristique des milieux forestiers méditerranéens, regroupe 57 mousses et 6 hépatiques. Pour chaque espèce, la chorologie et la fréquence selon le type de biotope sont précisées.

En Sicile, Raimondo, Dia et Cortini Pedrotti (1988) ont montré que la bryoflore se compose de 586 taxons infragénériques (475 mousses et 111 hépatiques), répartis en 166 genres de 65 familles. Les secteurs géographiques les mieux connus sont Messina (404 taxons et 89 références bibliographiques), Palermo (324 et 70), les monts Peloritani (338 et 60) et Catania (267 et 51 ).

Enfin, en Sardaigne, des prospections effectuées jusqu'à environ $1500 \mathrm{~m}$ d'altitude dans une grande partie de l'île (39 stations réparties sur les provinces de Sassari, Nuoro, Oristani et Cagliari), ont livré 182 taxons de mousses dont environ 22 sont nouveaux pour la Sardaigne (Cortini Pedrotti et Troiano 1985). L'écologie est précisée pour chacune de ces entités.

En ce qui concerne la Yougoslavie, Grgic (1985) a superficiellement fait le point sur les publications bryologiques consacrées à la Bosnie-Herzégovine depuis le milieu du siècle dernier.

Les travaux les plus récents contiennent des listes plus ou moins exhaustives de muscinées rencontrées dans les régions continentales de cette province (étages montagnard et subalpin des montagnes Igman et Bjelasnica: Grgic 1980 et 1982, forêts de Picea omorika (Pancic) Purkyne de la vallée de la Milesevka et de la montagne Veliki Stolac: Grgic 1983, sources thermales près de Bosanski Samac: Grgic 1984). D'autre part, Pavletic et Pulevic (1980), ont cité 21 espèces d'hépatiques, une espèce d'Anthocérote et 112 espèces de mousses récoltées sur le littoral, mais également sur les reliefs de l'intérieur du Monténégro.

Enfin, aucune contribution traitant de la bryoflore de l'Istrie et de la Macédoine yougoslave n'est parue durant la dernière décennie (Grgic, communication écrite). 
Pour l'Albanie, je ne dispose que d'une information limitée. Néanmoins, les travaux récents consacrés à ce pays semblent rares. Bischler, Jovet-Ast et Baudoin (1980) ont signalé 59 espèces d'hépatiques, dont 34 n'avaient pas encore été citées du territoire albanais. Les prospections de ces auteurs ont surtout été effectuées dans les zones côtières, mais également sur quelques contreforts montagneux, jusq'à $1150 \mathrm{~m}$ d'altitude. En dehors du catalogue floristique proprement dit, l'égologie des taxons (écarts altitudinaux, biotopes et formations végétales, substrat et $\mathrm{pH}$ du sol) a été étudiée en détail dans cet article.

Pour la Grèce, une abondante documentation est fournie par Preston (1984a et b). L'activité exploratoire, relativement importante au début de la décennie, semble s'être à présent ralentie, du moins provisoirement. Depuis la parution des travaux cités plus haut, Townsend (1986) a fourni une liste de 25 mousses, dont au moins 5 sont nouvelles sur le territoire hellénique.

Enfin, parmi les ouvrages ou publications importantes consacrés à la systématique et à la révision de certaines genres, on peut mentionner: Düll (1986: Rhynchostegiella en Europe et Macaronésie), Guerra et Ros (1987:Didymodon section Asteriscium en Péninsule Ibérique), Jovet-Ast (1986, Riccia: tout le bassin méditerranéen), Kramer (1980: Tortula section Rurales), Sergio (1982b: Ephemerum en Péninsule Ibérique).

B.- Les parties africaine et asiatique du bassin méditerranéen

Le sud et l'extrême est du bassin méditerranéen sont indiscutablement les parties les moins bien connues du point de vue bryofloristique. Ils constituent un immense territoire, inclus dans le projet de check-list des bryophytes du bassin méditerranéen d'O.P.T.I.M.A. (Organization for the Phytotaxonomical Investigation of the Mediterranean Area). Cet ambitieux projet nécessitera, outre un travail considérable de révision du matériel accumulé dans les herbiers, une intensifications de l'exploration bryologique de ces régions.

En ce qui concerne le Maroc, l'Algérie, la Tunisie et la Libye, aucune contribution bryofloristique importante n'a été publiée durant la dernière décennie, surtout faute de spécialistes résidant de façon permanente dans ces pays.

On en est donc resté à peu près au niveau de connaissance atteint en 1978 (cf. Hébrard 1982a). Toutefois, un article récent (Frahm 1988) énumère 23 mousses récoltées au Maroc et en Tunisie, mais également en Egypte [Didymodon australasiae (Hook. et Grev.) Zander est nouveau pour la Tunisie; Gymnostomum luisieri (Sergio) Sergio ex Crundw. et Tortula revolvens (Schimp.) G. Roth var. obtusata Reimers sont nouveaux pour la Tunisie et le Maroc].

En Egypte, où la bryoflore est plutôt pauvre (environ 130 mousses et 13 hépatiques), El Saadawi, Abou El Kheir et Darwish (1987) et El Saadawi, Badawi et Refai (1987) ont publié les résultats de leurs récoltes dans la région du delta du Nil. Les biotopes les plus riches sont situés à proximité immédiate du fleuve et des canaux 
d'irrigation, où l'humidité est importante durant une partie de l'année. Parmi les espèces intéressantes, on note: Brachymenium sp., Fissidens crassipes Wils. ex B., S. et G. var. philibertii Besch., Micropoma niloticum (Delile) Lindb., Philonotis hastata (Dub.) Wijk et Marg., Semibarbula orientalis (Web.) Wijk et Marg.

D'après la bibliographie contenue dans ces deux articles, on constate l'extrême rareté des travaux récents consacrés aux muscinées d'Egypte et des états voisins. Signalons à ce propos que Frey et Kürschner (1988), étudiant la distribution mondiale de Crossidium geheebii (Broth.) Broth., viennent de confirmer sa présence au Sinaï.

Pour la Jordanie, El Oqlah et Lahham (1985) ont cité quelques mousses du nord du pays, alors que Frey et Kürschner (1983) et El Oqlah, Frey et Kürschner (1988a) ont étudié en détail la bryoflore de Transjordanie. Au total, 110 mousses et 12 hépatiques sont mentionnées pour ce territoire (41 de ces bryophytes n'y avaient encore jamais été signalées), caractérisé par un pourcentage élevé de méditerranéennes au sens large («circum-tethyan element») et par quelques endémiques du ProcheOrient comme Grimmia mesopotamica Schiffn., Splachnobryum arabicum Dix. et Tortula rigescens Broth. et Geh.

Parmi les muscinées intéressantes, on remarque en particulier: Athalamia spathysii (Lindenb.) Hatt., Bryum syriacum Lor., Crossidium aberrans Holz. et Bartr., Funaria handelii Schiffn., Gymnostomum mosis (Lor.) Jur. et Milde, Riccia trabutiana Steph., Tortula brevissima Schiffn., Tortula revolvens (Schimp.) G. Roth. Ces travaux renferment également de précieuses informations relatives à la présence des espèces en Israël, au Liban, en Syrie et en Turquie.

En Israël, une flore complète illustrée des bryophytes est actuellement en préparation, sous la responsabilité d'I. Herrnstadt, qui a réalisé au préalable la révision critique de la majeure partie du matériel déposé dans l'herbier de l'université de Jérusalem (HUJ). Diverses régions du pays ont également été explorées. Ainsi, Herrnstadt, Heyn, Ben-Sasson et Crosby (1982) ont signalé 44 espèces de mousses nouvelles pour le territoire d'Israël, en particulier: Anoectangium handelii Schiffn., Cinclidotus pachyloma Salm., Didymodon aaronis (Lor.) Guerra, Fissidens arnoldii Ruthe, Gigaspermum mouretii Corb., Grimmia mesopotamica Schiffn., Tortula princeps De Not. subsp. echinata (Schiffn.) W. Kramer, Tortula handelii Schiffn. et Tortula pseudo-handelii Froehl.

Toutefois, beaucoup de résultats obtenus au cours des recherches effectuées sur le terrain pendant la dernière décennie n'ont pas encore été publiés.

Enfin, pour le Liban, Charouk (1982) a fourni les premiers éléments d'un inventaire bryofloristique de la vallée du Nahr Ibrahim (Liban central), depuis le littoral jusqu'à environ $1300 \mathrm{~m}$ d'altitude. Soixante six espèces de mousses et 21 espèces d'hépatiques ont été récoltées dans divers biotopes, le long de la vallée (rochers, sol et écorces d'arbres dans les ripisylves et les séries de Quercus calliprinos Webb et de Quercus infectoria Olivier).

Les bryophytes nouvelles pour le Liban sont les suivantes: Athalamia spathysii (Lindenb.) Hatt., Aulacomnium androgynum (Hedw.) Schwaegr., Calypogeia fissa 
(L.) Raddi, Cephaloziella baumgartneri Schiffn., Cephaloziella divaricata (Sm.) Schiffn., Cephaloziella stellulifera (Tayl. ex Spruce) Schiffn., Cephaloziella turneri (Hook.) K. Müll., Cinclidotus riparius (Brid.) Arnott, Jungermannia hyalina Lyell, Lophocolea heterophylla (Schrad.) Dum., Neckera besseri (Lob.) Jur., Orthotrichum diaphanum Brid., Orthotrichum limprichtii I. Hag., Polytrichum commune Hedw., Southbya nigrella (De Not.) Henriques, Tortella inflexa (Bruch) Broth., Weissia Brachycarpa (Nees et Hornsch.) Jur. et Weissia wimmerana (Sendtn.) B., S. et G.

\section{CHOROLOGIE}

Contrairement aux spermaphytes, beaucoup de bryophytes ont une aire géographique vaste. Dans la zone tempérée de l'hémisphère nord, de nombreuses espèces se retrouvent dans des biotopes favorables, en Europe, en Asie et en Amérique du Nord. En conséquence, à basse et moyenne altitude, du moins dans la région méditerranéenne d'Europe, les éléments circumboréal, méditerranéen au sens large (eury-et subméditerranéen, cf. Lecointe 1979, 1981 a et b) et méditerranéen-atlantique sont les mieux représentés.

Par contre, les muscinées «euméditerranéennes» ou méditerranéennesmacaronésiennes, n’ont généralement qu'une importance réduite, étant donné leurs faibles effectifs. Il s'agit de plus d'espèces rares, probablement archaïques, dont la répartition dans l'espace est souvent très limitée.

La liste ci-dessous énumère les principales mousses euméditerranéennes et méditerranéennes-macaronésiennes. Les abréviations des subdivisions géographiques (Afr 1, As 5) sont conformes à Düll (1984, 1985).

a) Mousses connues seulement du pourtour mésogéen (parfois d'un seul pays ou région):

Acaulon dertosense Casas, Cros, Sergio et Brugues (Hs), Anomobryum lusitanicum (I. Hag.) Thér. (Hs, Lu), Barbella strongylensis Bott. (Si), Cinclidotus pachylomoides Biz. (Gr, As 5), Fissidens kosaninii Latz. (Ga, Gr, Hs, It, Ju), Funaria maireana Copp. (Gr), Gigaspermum mouretii Corb. (Hs, Afr 1, As 5), Goniomitrium seroi Cas. Puig (Hs), Isothecium algarvicum Nicholson et Dix. (Hs, Lu), Mielichhoferia pontevedrensis Luis. (Hs, Lu), Phascum cuynetii Biz. et Pierr. (Hs), Pyramidula algeriensis Chudeau et Douin (Ga, Hs, Afr 1), Rhynchostegiella letourneuxii (Besch.) Broth. (Co, Ga, Hs, Lu, Afr 1), Thamnobryum cossyrense (Bott.) A.J.E. Sm. (Si), Triquetrella arapilensis Luis. (Hs, Lu), Vesicularia reimersiana Biz. et P. de la Varde (Si, Afr 1).

b) Mousses connues seulement du pourtour mésogéen (parfois d'un seul pays ou région) et se retrouvant dans l'ensemble macaronésien (Afr 5):

Entosthodon curvisetus (Schwaegr.) C. Müll. (Bl, Co, Cr, Ga, Gr, Hs, It, Ju, Lu, $\mathrm{Sa}, \mathrm{Si}$, Afr 1, As 5), Entosthodon pallescens Jur. (B1, Cr, Gr, Hs, It, Si, As 5), Fissidens 
ovatifolius Ruthe (Bl, Co, Cr, Ga, Hs, It, Ju, Lu, Sa, Si, Afr 1), Funaria convexa Spruce (Bl, Co, Cr, Ga, Gr, Hs, It, Lu, Sa, Si, Afr 1, As 5), Grimmia pitardii Corb. (Bl, Cr, Ga, Gr, Hs, It, Afr 1, As 5), Homalia lusitanica Schimp. (B1, Ga, Hs, It, Ju, Lu, Afr 1, As 5), Homalia webbiana (Mont.) Schimp. (Hs), Lepidopilum virens Card. (Hs), Neckera intermedia Brid. (Hs, Afr 1) ${ }^{(2)}$ Orthothecium durieui (Mont.) Besch. (Co, $\mathrm{Ga}, \mathrm{Hs}$, It, Sa, Afr 1), Ptychomitrium nigrescens (Kunze) Wijk et Marg. (Ga, Hs, It, Lu, Si), Scorpiurium sendtneri (Schimp.) Fleisch. (Cr, Gr, Hs, It, Ju, Lu, As 5), Sematophyllum bottinii (Breidl.) Podp. (Hs, It), Sematophyllum substrumulosum (Hampe) Britt. (Co, Ga, Hs, It, Ju, Lu, Afr 1).

Signalons que l'aire géographique totale de plusieurs de ces mousses et de trois hépatiques récemment découvertes dans la Péninsule Ibérique, a été étudiée en détail par Casas, Brugues et Cros (1981).

Dans le domaine de la chorologie, les publications de Düll (1983, 1984 et 1985) constituent, ainsi que nous l'avons vu, une source de documentation essentielle pour la totalité du bassin méditerranéen (la présence des taxons en Afrique du Nord et au Proche-Orient est mentionnée). Toutefois, les centres primaires de distribution utilisés dans ces ouvrages sont nombreux (environ 24), ce qui rend le système d'une utilisation mal aisée pour les synthèses, contrairement à celui que propose Lecointe (1979, 1981a et b, 1988). D'autre part, pour les hépatiques, Bischler et Jovet-Ast (1986) ont réalisé une synthèse englobant tout le sud-ouest asiatique: adaptation aux habitats, diversité spécifique, flores régionales, distribution mondiale de Marchantia paleacea Bertol., Plagiochasma rupestre (J.R. et G. Forst.) Steph., Riccia atromarginata Levier, Riccia gougetiana Durieu et Mont., Riccia huebeneriana Lindenb., Riccia lamellosa Raddi.

En ce qui concerne la cartographie des espèces, rien n'a encore été entrepris, à ma connaissance, en Afrique du Nord et au Proche-Orient. Il n'en est pas de même en Europe méridionale, où les progrès accomplis dans ce domaine durant la dernière décennie sont considérables. Pour la Péninsule Ibérique, hormis l'important recueil publié par Casas, Brugues et Cros (1985), des cartes de répartition plus ou moins précises sont disponibles pour d'assez nombreuses muscinées (cf. entre autres: Casas, Simo et Varo 1981, Casas et al. 1988, Casas de Puig 1979, Cros et Rosselló 1984, Elias Rivas 1988, Fuertes Lasala et Acon Remacha 1988, Gil et Castro 1984, Gil García et Castro 1987, Guerra et Gil 1981, 1982, Rosselló 1981, Sergio 1980, 1982b, 1988 etc...). Par contre, des documents récents de ce type manquent encore pour la France méditerranéenne, la Sardaigne, la côte yougoslave et albanaise et la Grèce. Ils sont rares pour l'Italie méditerranéenne [Dia 1987: Ulota crispa (Hedw.) Brid., Raimondo et Dia 1979: Cryphaea heteromalla (Hedw.) Mohr, Schumacker et al. 1988: Amblystegium compactum (C. Müll.) Aust.] et la Corse [Hébrard 1984a: cartographie d'Anacolia webbii (Mont.) Schimp., d'Entosthodon curvisetus (Schwaegr.) C. Müll. et de Grimmia tergestina Tomm. ex B., S. et G., UTM 10 x $10 \mathrm{Km}]$.

En 1980 a été créé le groupe de travail pour la cartographie des bryophytes en Europe (secrétariat: R. Schumacker, université de Liège, Belgique). Le projet initial 
était de cartographier environ 200 mousses et 170 hépatiques, en utilisant la grille de l'atlas de Flora Europaea (UTM 50 x $50 \mathrm{Km}$ ). En novembre 1988, le nombre de cartes achevées (ou presque) s'élevait à 65 pour les mousses et à 59 pour les hépatiques. Parmi ces bryophytes figurent d'une part des espèces présentes ailleurs en Europe, mais répandues aussi au sud de $45^{\circ}$ de latitude nord [hépatiques: Cololejeunea minutissima (Sm.) Schiffn., Lophocolea fragrans (Moris et De Not.) Gott. et al., Marchesinia mackaii (Hook.) S. Gray, Saccogyna viticulosa (L.) Dum.; mousses: Crossidium squamiferum (Viv.) Jur., Epipterygium tozeri (Grev.) Lindb., Fissidens algarvicus Solms], et d'autre part des muscinées presque exclusivement confinées à la partie méridionale du continent, telle que nous venons de la définir [hépatiques: Anthoceros mandonii Steph., Asterella africana (Mont.) Evans, Frullania illyrica Grolle, Plagiochasma rupestre (J.R. et G. Forst.) Steph.; mousses: Anacolia webbii (Mont.) Schimp., Antitrichia californica Sull., Cheilothela chloropus (Brid.) Lindb., Crossidium crassinerve (De Not.) Jur., Entosthodon curvisetus (Schwaegr.) C. Müll., Gigaspermum mouretii Corb., Goniomitrium seroi Cas. de Puig, Gymnostomum luisieri (Sergio) Sergio ex Crundw., Homalothecium aureum (Spruce) Robins., Oedipodiella australis (Wager et Dix.) Dix., Orthothecium durieui (Mont.) Besch., Sematophyllum substrumulosum (Hampe) Britt., Triquetrella arapilensis Luis.].

\section{ÉTUDE DES COMMUNAUTÉS BRYOPHYTIQUES MÉDITERRANÉENNES}

\section{A - Bryophytosociologie et écologie}

En Péninsule Ibérique, la bryophytosociologie a connu, durant la dernière décennie, un essor considérable.

Dans le sud de l'Espagne notamment, les résultats obtenus grâce à l'utilisation de la méthode zuricho-montpelliéraine, comblent des lacunes importantes, puisqu'ils traitent de l'étage subhumide et surtout du semiaride. En ce qui concerne le semiaride, Ros et Guerra (1987b), dans un ouvrage exhaustif consacré à la végétation bryophytique terricole du sud-est de l'Espagne et à l'écologie des communautés (en particulier physico-chimie du sol), ont décrit par exemple l'ordre des Tortulo brevissimae Aloinetalia bifrontis [Aloina bifrons (De Not.) Delgadillo, Crossidium crassinerve (De Not.) Jur., Didymodon aaronis (Lor.) Guerra, Tortula brevissima Schiffn., Riccia crustata Trab. ex Grolle], qui regroupe des unités basophiles réparties pour le moment en deux alliances. A l'étage subhumide, Gil et Guerra (1981) et Guerra (1982a) ont étudié les bryoassociations épiphytes des sierras d'Algeciras et des pinsapares d'Andalousie, alors que Gil et León (1984) ont décrit le Distichio capillaceiAthalamietum hyalinae, propre aux fissures de rochers calcaires ou dolomitiques, dont l'optimum se situe aux alentours de 1800 m d'altitude, sur les chaînes bétiques.

Liste simplifiée des principales unités bryophytosociologiques décrites dans la Péninsule Ibérique durant la dernière décennie. 
- Unités rupicoles

- Terrains siliceux

Hedwigion ciliatae Philippi 1956

$\checkmark$ Communauté à Hedwigia ciliata et Grimmia trichophylla Guerra 1982

- Terrains calcaires

Ctenidion mollusci Stefureac 1941

$\checkmark$ Distichio capillacei-Athalamietum hyalinae Gilet León 1984

- Unités terricoles

- Terrains siliceux et (ou) acides

Dicranellion heteromallae Philippi 1966

$\diamond$ Fossombronio angulosae- Phaeoceretum bulbiculosi Guerra, Gil et Varo 1981

$\checkmark$ Saccogyno viticulosae-Fissidentetum serrulati Guerra, Gil et Varo 1981

$\checkmark$ Calypogeio fissae-Pallavicinietum lyellii Guerra, Gil et Varo 1981

Campylopodion fragili-introflexi Guerra, Gil et Varo 1981

o Polytricho-Campylopodetum polytrichoidis Guerra, Gil et Varo 1981

Ceratodo-Polytrichion piliferi Smarda 1947

$\diamond$ Communauté à Polytrichum piliferum et Bartramia stricta Guerra 1982

- Terrains calcaires et (ou) basiques

Pleurochaetion squarrosae Neumayr 1971

$\diamond$ Homalothecio aurei-Pleurochaetetum squarrosae Ros et Guerra 1987

$\checkmark$ Communauté à Homalothecium aureum et Scleropodium touretii Ros et Guerra 1987

Cephaloziello-Southbyion nigrellae Guerra et Gil 1982

$\diamond$ Gymnostomo luisieri-Southbyetum nigrellae (Guerra et Gil 1982) Ros et Guerra 1987

Tortulo brevissimae-Aloinetalia bifrontis Ros et Guerra 1987

Aloino-Crossidion crassinervis Ros et Guerra 1987

$\checkmark$ Crossidio crassinervis-Tortuletum obtusatae Ros et Guerra 1987

$\checkmark$ Acaulo triquetri-Tortuletum brevissimae Ros et Guerra 1987

Pottio-Riccion crustatae Ros et Guerra 1987

$\diamond$ Riccietum crustatae Ros et Guerra 1987

○ Crossidio crassinervis-Pottietum commutatae Ros et Guerra 1987

- Autres types de substrats

Tortellion flavovirentis Guerra 1982

$\checkmark$ Trichostomo littoralis-Orthothecietum durieui Guerra 1982

$\checkmark$ Tortello-Bryetum dunensis Guerra et Puche 1984

Phascion mitraeformis Waldheim 1947

$\diamond$ Riccietum atromarginato-lamellosae Ros et Guerra 1987

Mannion androgynae Ros et Guerra 1987

$\checkmark$ Riccio nigrellae-Oxymitretum paleaceae Ros et Guerra 1987

- Unités corticoles

Fabronion pusillae Barkman 1958

$\checkmark$ Pterigynandro-Orthotrichetum speciosi Guerra 1982

Ulotion crispae Barkman 1958

$\diamond$ Orthotricho-Neckeretum pumilae Guerra 1982

$\checkmark$ Neckero laevifoliae-Porelletum canariensis Gil et Guerra 1981

Antitrichion curtipendulae (Ochsner 1928) Barkman 1958

$\checkmark$ Antitrichio-Isothecietum algarvici Guerra 1982 
Dans le midi de la France et en Corse, la méthode sigmatiste a également été employée pour l'étude de certains groupements. Une large part a été faite à l'écologie (édaphisme en particulier), au détriment de l'aspect purement synsystématique.

En effet, étant donné la multiplication exagérée des unités bryophytosociologiques, en rapport avec des différences d'appréciation et de méthodologie selon les auteurs, peu de tentatives ont été faites dans ces régions pour situer les groupements.

Pour le sud-est français, les principaux travaux traitent des pinèdes de pin d'Alep sur calcaire (Hébrard 1984b: végétation bryophytique et pédologie dans les parties basses des Bouches-du-Rhône et du Var), des communautés muscinales des troncs de Quercus ilex L. et de Quercus pubescens Willd. dans le département du Var (Hébrard 1987c: utilisation de la dominance mesurée des bryophytes pour définir les communautés occupant la base des troncs), de l'écologie et de la sociologie d'espèces rares dans ces départements (Hébrard 1982b). Enfin, l'étude des variations des valeurs du pH du substrat a été réalisée pour 8 mousses de l'île de Port-Cros (Hébrard 1979).

Dans la région basse de la Corse, plusieurs contributions concernent divers types de pelouses méditerranéennes sur silice [Hébrard 1980: groupements à Entosthodon fascicularis (Hedw.) C. Müll. et Pleuridium acuminatum Lindb., à Phascum cuspidatum Hedw. et Pottia sp. pl., à Riccia gougetiana Durieu et Mont. et Riccia ciliata Hoffm.], les forêts de Quercus ilex L., de Quercus suber L. ainsi que les maquis bas du Cap Corse (Hébrard 1981), l'écologie et la sociologie d'Anacolia webbii (Mont.) Schimp., d'Entosthodon curvisetus (Schwaegr.) C. Müll. et de Grimmia tergestina Tomm. ex B., S. et G. (Hébrard 1984a).

En Italie, les travaux bryophytosociologiques réalisés au cours de la dernière décennie sont en majeure partie consacrés à la Sicile (cf. Cortini Petrotti 1986).

Ils traitent notamment des groupements calciphiles tufogènes (Raimondo, Dia et Rossitto 1981, Privitera et Lo Giudice 1986), de l'Hedwigion ciliatae Philippi 1956 (Dia 1985), ou encore de la végétation muscinale de certaines hêtraies des monts Peloritani [Privitera et Lo Giudice 1981; végétation rupicole: groupement à Thamnobryum alopecurum (Hedw.) Nieuwl., association à Grimmia pulvinata (Hedw.) Sm. et Tortula muralis Hedw. Von Hübschmann 1950; végétation aquatique: Oxyrrhynchietum rusciformis Gams. 1927; végétation des écorces d'arbres: groupement à Isothecium myurum Brid. et Homalothecium sericeum (Hedw.) B., S. et G., Orthotrichetum lyellii Lecointe 1975] et des unités corticoles à Cryphaea heteromalla (Hedw.) Mohr (Raimondo et Dia 1979).

Les études les plus récentes concernent des territoires de superficie réduite. Ainsi, les forêts de Quercus ilex L., de Quercus suber L., et certaines cistaies, qui occupent à basse altitude des sols détritiques plus ou moins décalcifiés, dans la région de Santo-Pietro (Sicile méridionale), ont été prospectées par Privitera et Lo Giudice (1985). Les conditions édaphiques, les préférences écologiques des muscinées, ainsi que leur place dans la dynamique de la végétation forestière ont été prises en considération. 
Dans l'Enna (Lo Giudice et Privitera 1987a), sur les calcarénites et argiles du vallone Murapana, entre 400 et $600 \mathrm{~m}$ d'altitude, ont été reconnus l'OrthotrichoGrimmietum pulvinatae (Gams 1927) Stodiek 1937 (rupicole), trois bryoassociations tufogènes, un groupement terricole à Trichostomum brachydontium Bruch, ainsi que l'Anomodonto-Isothecietum vivipari Lippmaa 1935 (association corticole sur Populus et Salix).

Enfin, dans la grotte Garonne, près de Palerme, Lo Giudice et Privitera (1987b) ont mis en évidence la zonation de bryophytes selon l'intensité de l'éclairement. Douze relevés effectués sur les rochers calcaires et les parois de cette cavité, ont amené les auteurs à discuter la validité du Thamnobryetum alopecuri Gams 1927, unité hétérogène et écologiquement mal définie.

En Yougoslavie, quelques travaux récents employant la méthode zurichomontpelliéraine ont été consacrés aux groupements muscinaux présents dans certains massifs calcaires de Bosnie-Herzégovine, où les influences climatiques méditerranéennes sont déjà très atténuées.

Ainsi, aux étages montagnard et subalpin des montagnes Igman et Bjelasnica, et notamment en milieu forestier, les bryoassociations rupicoles, terricoles, corticoles et lignicoles ont été étudiées par Grgic (1980 et 1982). Cet auteur a décrit entre autres l'Encalypto (streptocarpae) -Eurhynchietum strigosi Grgic 1980 (terricole dans l'Aceri obtusati-Fagetum) et le Pseudoleskeetum filamentosaeGrgic 1982 [sur l'écorce des troncs de Fagus moesiaca (K. Maly) Czecz.].

Enfin, le problème de l'utilisation des bryophytes épiphytes comme indicateurs de la qualité de l'air dans l'agglomération de Sarajevo a été abordé par Grgic et Puhar (1987).

Dans tous les autres pays du bassin méditerranéen, la bryophytosociologie et à plus forte raison l'écologie se trouvent encore à un stade rudimentaire.

Deux contributions phytosociologiques consacrées aux forêts des étages supraméditerranéen et montagnard d'Epire, de Macédoine grecque et de Thrace, prennent en compte les muscinées (groupements terricoles et épiphytes: Gamisans et Hébrard 1979, 1980), alors qu'au Liban Charouk (1982) a étudié la distribution des bryophytes en fonction des caractères physico-chimiques du substrat, de la zonation altitudinale et des étages de végétation.

Enfin, quelques synthèses limitées à des thèmes précis traitent de l'ensemble du bassin méditerranéen. Tel est le cas pour Baudoin, Bischler et Hébrard (1987: hépatiques de la pinède méditerranéenne de Pinus halepensis Miller) et Baudoin, Bischler, Jovet-Ast et Hébrard (1984: banque de données phytoécologiques des hépatiques de la région méditerranéenne).

B - Dynamique des communautés bryophytiques méditerranéennes

1 - Dynamique du peuplement bryophytique des certaines forêts de chêne vert et de chêne pubescent dans le département du Var (France). 
Ces recherches ont été effectuées dans des taillis de chêne vert (Hébrard et Rolando 1985), ou de chêne pubescent (Hébrard 1987b) d'âge connu (quatre classes d'âge de 6-11 ans à 61-66 ans), sur le site de la Gardiole de Rians, en terrain calcaire.

Outre la structure des communautés bryophytiques épigées, on a étudié, en utilisant des méthodes assez comparables à celles de Slack (1977), la variation dans le temps du recouvrement muscinal mesuré, mais également de la fréquence et de la dominance mesurée de chaque espèce de mousse ou d'hépatique.

Par exemple, dans les taillis de chêne vert, la valeur du recouvrement muscinal au sol, toujours très faible (effet litière), varie avec l'exposition des stations et diminue lorsque l'âge des taillis augmente.

En outre, la composition de la communauté bryophytique présente à la fois sur le sol proprement dit et la roche affleurante, tend à s'uniformiser au fur et à mesure du vieillissement des taillis.

2 - Banques édaphiques de diaspores et stratégies de vie adaptatives des bryophytes dans les écosystèmes méditerranéens.

Tout récemment, During, Brugues, Cros et Lloret (1987) ont étudié les banques édaphiques de diaspores (bryophytes et ptéridophytes) de différentes formations végétales dans le région de Barcelone (formations à Chamaerops humilis L., à Rosmarinus officinalis L. et Gypsophila hispanica Willk., pinède, chênaie pubescente et même hêtraie).

Par exemple, dans la chênaie pubescente de Sant Llorenç, à environ $800 \mathrm{~m}$ d'altitude, la banque édaphique de diaspores se compose d'un fort pourcentage d'espèces colonisatrices, dont certaines sont absentes de la végétation bryophytique épigée de la placette dans laquelle le sol a été prélevé.

D'autre part, sur l'ensemble des sites, le type «colonisateur» est le plus répandu dans le sol, avec plus localement des espèces appartenant aux types «annuel» ou «itinérant à vie courte» (cf. During 1979). Le type «colonisateur» est donc à peu près omniprésent dans le sol sous forme de diaspores, y compris dans les stations où la végétation bryophytique est presque exclusivement constituée de Pleurocarpes pérennes.

Il ressort de ces intéressants travaux, que le sol d'un écosystème donné renferme une réserve de diaspores de bryophytes, susceptible de contribuer à la régénération d'un couvert muscinal différent, lorsque la végétation en place est détruite ou profondément altérée (incendie, coupe). Ceci pose évidemment le problème de la longévité et de la durée de conservation des diaspores. Pour les bryophytes des milieux méditerranéens, ce champ d'investigations est important.

\section{CONCLUSION}

L'analyse succincte des apports récents (1979-1988) dans le domaine de la bryologie méditerranéenne, met en évidence une répartition très inégale de l'activité 
entre la partie européenne du bassin méditerranéen, où les bryologues autochtones sont relativement nombreux, et enfin les pays du Maghreb où ils manquent. Ainsi, des check-lists (mousses et hépatiques) couvrant l'ensemble du continent européen ont été publiées durant la période considérée. Toutefois, de telles synthèses n'ont pas encore été effectuées, à plus grande échelle, pour le sud de la France et la Corse, l'Italie péninsulaire, la Yougoslavie et l'Albanie. Malgré l'importance des travaux taxonomiques réalisés, notamment en Péninsule Ibérique, peu d'espèces nouvelles pour la science ou pour le continent ont été découvertes dans le sud de l'Europe.

Par contre, compte tenu de l'essor qu'a connu l'exploration bryofloristique en Péninsule Ibérique, mais également dans le midi de la France, en Corse, en Italie, en Sardaigne, en Sicile et en Grèce, un certain nombre de muscinées, jusqu'à présent non encore citées de ces pays, y ont été signalées postérieurement aux travaux de Düll (1983, 1984 et 1985; Corse: 4 mousses, Espagne: 1 hépatique et 4 mousses, France sud: 4 mousses, Grèce: 5 mousses, Portugal: 5 hépatiques et 8 mousses, Sardaigne: 5 mousses, Sicile: 3 mousses).

D'autre part, la création dès 1980 du groupe de travail pour la cartographie des bryophytes en Europe, basé sur la coopération internationale, a permis d'établir environ 124 cartes de répartition de muscinées, dont certaines sont fréquentes en région méditerranéenne.

En ce qui concerne la sociologie et l'écologie des bryophytes, l'apport des auteurs espagnols est considérable, en particulier du point de vue synsystématique. Néanmoins, des travaux ont également été réalisés dans ces domaines en France méridionale en en Corse, en Grèce, en Italie et en Sicile, ainsi qu'au Portugal et en Yougoslavie.

Plus récemment ont été abordés divers aspects de la dynamique des communautés muscinales: structure des communautés muscinales de forêts de chêne vert et de chêne pubescent d'âge différent en Provence, étude des banques édaphiques de diaspores de bryophytes de plusieurs formations végétales en Catalogne espagnole.

Dans le reste du bassin méditerranéen, les travaux publiés traitent essentiellement de floristique. Durant la période considérée, l'exploration bryofloristique a été poursuivie activement en Egypte, en Israël et en Jordanie. Par contre, les pays du Maghreb n'ont pas fait l'objet d'études récentes importantes et offrent aux bryologues un champ immense d'investigations.

\section{REMERCIEMENTS}

J'adresse mes plus vifs remerciements au comité d'organisation du «VIII simposios de ciencias criptogámicas», qui m'a invité à participer à ce congrès, en prenant en charge mes frais de déplacement et de séjour à Melilla.

Je remercie également tous mes collègues bryologues qui, au cours des années, m'ont envoyé leurs travaux, contribuant ainsi à la documentation de cet exposé. R. 
Schumacker (station scientifique des Hautes Fagnes, université de Liège) a mis à ma disposition des copies de cartes de répartition de bryophytes en Europe et m'a très aimablement autorisé à en utiliser quelques unes. C. Cortini Pedrotti (université de Camerino), M. Privitera et R. Lo Giudice (université de Catania), ainsi que P. Grgic (université de Sarajevo), m'ont fourni de la documentation récente concernant respectivement la totalité du territoire italien, la Sicile et le sud de la Yougoslavie.

\section{BIBLIOGRAPHIE}

ANDREANI, L. \& C. CORTINI PEDROTTI - 1986- Flora briologica della selva dell' Abbadia di Fiastra nelle Marche (Italia). Webbia, 39(2):281-288, 2 tabl., 3 fig.

ARTS, T. -1988- The occurence of drought - resistant rhizoidal tubers in Haplodontium notarisii. Lindbergia, 14(2):131-132, 1 fig.

ARTS, T. \& G. NORDHORN-RICHTER -1986- Epipterygium tozeri in Europe, its distribution and vegetative propagation. Journ. of Bryology, 14(1):91-97, 4 fig.

BAUDOIN, R., H. BISCHLER \& J.P. HÉBRARD -1987- Les hépatiques de la pinède méditerranéenne de Pinus halepensis Miller. Symposia Biologica Hungarica, 35:389-404, 1 tabl., 6 fig.

BAUDOIN, R., H. BISCHLER, S. JOVET-AST \& J.P. HÉBRARD -1984- Une banque de données phytoécologiques des hépatiques de la région méditerranéenne (Bryomed). Webbia, 38:385-396, 9 fig.

BISCHLER, H. \& S. JOVET-AST -1986- The hepatic flora of South-West Asia: A survey. Proc. Roy. Soc. Edinburgh, 89B:229-241, 13 fig.

BISCHLER, H., S. JOVET-AST, \& R. BAUDOIN -1980- Hépatiques de la côte albanaise. Cryptogamie, Bryol. Lichénol, 1(3):247-267, 2 tabl., 1 fig.

BLOCKEEL, T.L. \& A.C. CRUNDWELL - 1987- New bryophyte records from the Balearic Islands. Journ. of Bryology, 14(3):519-522.

CASAS, C. -1985- Rhodobryum ontariense (Kindb.) Kindb. a Catalunya. Orsis, 1:3-7, 3 fig.

CASAS, C., M. BRUGUES \& R.M. CROS -1981- Contribucio al coneixement de l'àrea geogràfica d'alguns briòfits. Treb. Inst. Cat. Hist. Nat., 9:169-178, 11 fig.

CASAS, C., M. BRUGUES \& R.M. CROS -1984- Referencies bibliogràfiques sobre la flora briològica hispanica II. Treb. Inst. Bot. Brcelona, 9:5-24.

CASAS, C., M. BRUGUES \& R.M. CROS -1985-Bryophytes cartography: Iberian Peninsula. Balearic and Canary Islands, Azores and Madeira. fasc. 1, 1-50, 154 pp. Barcelona: Institut d'Estudis Catalans.

CASAS, C., R.M. SIMO \& J. VARO -1981- Aportaciones al conocimiento de la flora briológica española. Notula V: Avance sobre un estudio de la Sierra de la Demanda. An. Jard. Bot. Madrid, 37(2):431-454, 10 fig.

CASAS, C., P. HERAS, J. REINOSO \& J. RODRIGUEZ-OUBIÑA - 1988- Consideraciones sobre la presencia en España de Campylopus introflexus (Hedw.) Brid. y C. pilifer Brid. Orsis, 3:21-26, 2 fig.

CASAS, C., C. SERGIO, R.M. CROS \& M. BRUGUES -1986- Acaulon dertosense sp. nov., musgo terrícola de los olivares del Baix Ebre (Cataluña). An. Jard. Bot. Madrid, 42(2):299301, 1 fig.

CASAS DE PUIG, C. -1979- Funaria pallescens (Jur.) Broth. var. mitratus (Cas. Gil) Wijk et 
Marg. en Menorca. Rev. Bryol. et Lichénol., 45(4):467-470, 2 fig.

CASAS SICART, C. -1981- The mosses of Spain, an annotated check-list. Treb. Inst. Bot. Barcelona, 7:1-57.

CASAS SICART, C. -1988- Datos para la brioflora de la Sierra de Gredos. Lazaroa, 10(19.87):265-267.

ÇETIN, B. -1988a-Check-list of the liverworts and hornworts of Turkey. Lindbergia, 14(1):1214.

ÇETIN, B. -1988b- Check-list of the mosses of Turkey. Lindbergia, 14(1):15-23.

ÇETIN, B. -1988c-Cinclidotus nyholmiae, a new species from Köprülü Canyon national park (Antalya) in Turkey. Journ. of Bryology, 15(2):269-273, 1 tabl., 2 fig.

CHAROUK, S. -1982- Contribution à l'étude bryologique du Liban. Mousses et hépatiques de la vallé de Nahr Ibrahim. Thèse Doctorat 3eme cycle, Université de Droit, d'Economie et des Sciences, Marseille, $110 \mathrm{p}$.

CORLEY, M.F.V., A.C. CRUNDWELL, R. DÜLL, M.O.HILL \& A.J.E. SMITH -1982Mosses of Europe and the Azores; an annotated list of species, with synonyms from the recent literature. Journ. of Bryology, 11(4)(1981):609-689.

CORTINI PETROTTI, C. -1983- Prodromo dei muschi della Sardegna. Lavori della Societa Italiana di Biogeografia, 8(1980):139-169.

CORTINI PEDROTTI, C. -1985- La florule bryologique des collines sablonneuses du lac Trasimène (Ombrie), Cryptogamie, Bryol. Lichénol, 6(1):59-63.

CORTINI PEDROTTI, C. -1986- Bibliografia briologica d'Italia. Webbia, 39(2):289-353.

CORTINI PEDROTTI, C. \& U. TOSCO -1988- Cento anni di briologia in Italia. In: 100 anni di ricerche botaniche in Italia (1888-1988). Firenze: Societa botanica italiana, p.p. 407424, 3 fig.

CORTINI PEDROTTI C. \& R. TROIANO - 1985-Contributo alla conoscenza dei muschi della Sardegna. Boll. Soc. Sarda Sc. Nat., 24:123-147, 1 fig.

CROS, R.M. \& J.A. ROSSELLÓ -1984- An approach to the bryophytic flora of the Pityusic Islands. In Biogeography of the Pityusic Islands, Kuhbier H., Alcover J.A. et Guerau d'Arellano Tur J.A. éd. The Hague, Boston, Lancaster: W. Junk, p.p. 155-170, 9 fig.

DANIN, A. \& M.G. BARBOUR -1982- Microsuccession of cryptogams and phanerogams in the Dead Sea area, Israel. Flora, 172:173-179.

DIA, M.G. -1985- Briocenosi dell'Hedwigion ciliatae Philippi 1956 rilevata in Sicilia. Inform. Bot. Ital. (Boll. Soc. Bot. Ital.), 15(2-3)(1983):172-173.

DIA, M.G. -1987- Note briogeografiche. V. Distribuzione ed ecologia di Ulota crispa (Hedw.) Brid. in Italia. Cryptogamie, Bryol. Lichénol. 8(3):241-250, 1 tabl., 1 fig.

DIA, M.G., G. MICELI \& R. NOT -1985- Check-list delle epatiche note in Sicilia. Webbia, 39(1):163-177.

DIA, M.G., G. MICELI \& F.M. RAIMONDO -1987- Check-list dei muschi noti in Sicilia. Webbia, 41(1):61-123.

DÜLL, R. -1983- Distribution of the european and macaronesian liverworts (Hepaticophytina). Bryologische Beiträge, 2:1-114.

DÜLL, R. -1984- Distribution of the european and macaronesian mosses (Bryophytina) Part I. Bryologische Beiträge, 4:1-113.

DÜLL, R. -1985- Distribution of the european and macaronesian mosses (Bryophytina) Part II. Bryologische Beiträge, 5:110-232.

DÜLL, R. -1986- Revision of Rhynchostegiella and closely related taxa in Macaronesia with reference to their occurence in Europe. Bryologische Beiträge, 6:91-105, 8 fig. 
DURING, H.J. - 1979- Life strategies of bryophytes: a preliminary review. Lindbergia, 5(1):218,1 tabl., 6 fig.

DURING, H.J., M. BRUGUES, R.M. CROS \& F. LLORET -1987- The diaspore bank of bryophytes and ferns in the soil in some contrasting habitats around Barcelona, Spain. Lindbergia, 13(3):137-149, 8 tabl.

ELIAS RIVAS, M.J. -1988- Comentarios sobre algunos táxones interesantes de la brioflora ibérica. Cryptogamie, Bryol. Lichénol., 9(4):353-362, 7 fig.

EL OQLAH, A.A. \& J.N. LAHHAM - 1985- Mosses from northern part of Jordan. Candollea, 40:147-152.

EL OQLAH, A.A., W. FREY \& H. KÜRSCHNER -1988a- The bryophyte flora of Transjordan. A catalogue of species and floristic elements. Willdenowia, 18:253-279, 1 tabl., 3 fig.

EL OQLAH A.A., W. FREY \& H. KÜRSCHNER -1988b- Tortula rigescens Broth. et Geh. (Pottiaceae), a remarkable species new to the moss flora of Jordan. Lindbergia, 14(1):2729, 1 fig.

EL-SAADAWI, W.E., W.S. ABOU EL-KHEIR \& M.H. DARWISH - 1987- Notes on egyptian mosses with one new record. Lindbergia, 12(2-3) (1986):129-131, 2 tabl., 1 fig.

EL-SAADAWI, W.E., A.A. BADAWI \& M.S. REFAI -1987- Mosses of the Nile delta. Lindbergia, 12(2-3) (1986):106-110, 3 tabl., 1 fig.

FRAHM, J.P. -1988- Bemerkenswerte Laubmoosfunde aus Nordafrika. Cryptogamie, Bryol. Lichénol., 9(3):231-234.

FREY, W. \& H. KÜRSCHNER - 1983- New records of bryophytes from Transjordan with remarks on phytogeography and endemism in SW asiatic mosses. Lindbergia, 9(2):121132, 5 fig.

FREY, W. \& H. KÜRSCHNER -1988- Re-evaluation of Crossidium geheebii (Broth.) Broth. (Pottiaceae) from Sinai, a xerothermic pangaean element. Journ. of Bryology, 15(1):123126,2 fig.

FUERTES LASALA, E. -1988- Notas briosociológicas de la Península Ibérica. I. Ulotetum crispae Ochsner 1928. Studia Botanica, 7:215-218, 1 tabl., 1 fig.

FUERTES LASALA, E. \& M. ACON REMACHA -1988- Cartografía de briófitos. II. Dumortiera hirsuta $(\mathrm{Sm}$.) Nees, Grimmia torquata Grev. y Cirriphyllum tenuinerve (Lindb.) Wijk. Studia Botanica, 7:209-214, 3 fig.

GAMISANS J. \& J.P. HÉBRARD -1979- A propos de la végétation des forêts d'Epire et de Macédoine grecque occidentale. Doc. Phytosoc. Lille, 4:289-341, 14 tabl., 1 fig.

GAMISANS, J. \& J.P. HÉBRARD -1980- A propos de la végétation en Grèce du Nord-Est (Macédoine orientale et Thrace occidentale). Doc. Phytosoc. Lille, 5:243-289, 11 tabl.

GAUTHIER, R. -1987- Note sur trois mousses du Sahara algérien. Cryptogamie, Bryol. Lichénol., 8(1):27-29.

GIL, J.A. \& J. CASTRO - 1984- Contribución al conocimiento de la brioflora de Sierra Morena (Jaén). Collect. Bot., 15:249-258, 6 cartes.

GIL J.A. \& J. GUERRA -1981- Aportaciones briosociológicas ibéricas. I. Comunidades epifitas de las Sierras de Algeciras. An. Jard. Bot. Madrid, 37(2):703-719, 3 tabl., 1 fig.

GIL, J.A. \& J. GUERRA - 1985- Estudio briosociológico de las Sierras de la Demanda y Urbión (España). Cryptogamie, Bryol. Lichénol., 6(3):219-258, 19 tabl., 3 fig.

GIL, J.A. \& G. LEON -1984- Distichio capillacei-Athalamietum hyalinae ass. nova en las cordilleras béticas. Fol. Bot. Misc., 4:113-120, 1 tabl., 3 fig.

GIL GARCIA, J.A. \& J. CASTRO -1987- Datos brioflorísticos sobre el sector marianicomonchiquense (Península Ibérica). Acta Bot. Malacit., 12:67-80, 22 cartes. 
GRGIC, P. -1980- The phytocoenoses of the bryophyta on the vertical profile of the mountains Igman and Bjelasnica. I. The epilithic and terrestrial vegetation. Godis. Biol. Inst. Univ. Sarajevo, 33:59-85, 4 tabl., 3 fig.

GRGIC, P. -1982- The phytocoenoses of the bryophyta on the vertical profile of the mountains Igman and Bjelasnica. II. The epiphytic and ligniphilic vegetation. Godis. Biol.Inst. Univ. Sarajevo, 35:47-69, 5 tabl., 6 fig.

GRGIC, P. -1983- A contribution to the knowledge of mosses in the ecosystems with the serbian spruce. Godis. Biol. Inst. Univ. Sarajevo, 36:73-78.

GRGIC, P. -1984- Epilithic community of moss in the wells of the nephropathic region in the vicinity of Bosanski Samac. Bilten Drustva Ekologa Bosne I. Hercegovine ser.B, 2:311 314, 1 tabl.

GRGIC, P. -1985- The extent and characteristics of bryophyta investigations in Bosnia and Herzegovina. Godis Biol. Inst. Univ. Sarajevo, 38:33-41.

GRGIC, P. \& R. LAKUSIC -1987- Pioneer plant communities on the ash heap disposals. Godis. Biol. Inst. Univ. Sarajevo, 40:17-25, 1 tabl.

GRGIC, P. \& S. PUHAR -1987- A contribution to the knowledge of the epiphytic vegetation as an indicator of air quality in Sarajevo and its environment. Bilten Drustva Ekologa Bosne I Hercegovine, ser. A, 4:135-146, 1 tabl.

GROLLE, R. -1983- Hepatics of Europe including the Azores: an annotated list of species, with synonyms from the recent literature. Journ. of Bryology, 12(3):403-459.

GUERRA, J. -1982a- Vegetación briofítica epifita del dominio climácico de Abies pinsapo Boiss. Cryptogamie, Bryol. Lichénol. 3(1):9-27, 4 tabl., 3 fig.

GUERRA, -1982b- Estudio briofítico de los macizos serpentínicos de Sierra Bermeja y Sierra de Aguas (Málaga, España). Acta Bot. Malacit., 7:151-171, 5 tabl., 1 fig.

GUERRA, J. \& J.A. GIL-1981- Aportaciones a la flora briofítica de Andalucía. I. Trab. Monogr. Depto. Bot. Málaga, 2:13-26, 1 fig., 4 cartes.

GUERRA, J. \& J.A. GIL -1982- Comunidades briofíticas mediterráneas de protosuelos calcáreos húmedos. Fol. Bot. Misc., 3:87-94, 2 tabl., 1 fig.

GUERRA, J. \& R.M. ROS -1987- Revisión de la sección Asteriscium del género Didymodon (Pottiaceae, Musci) en la Península Ibérica. Cryptogamie, Bryol. Lichénol., 8(1):47-68, 2 graphiques, 3 fig., 3 cartes, 2 pl. phot.

GUERRA, J. \& R.M. ROS -1988- Caracterización taxonómica, corología y ecología de Pottia pallida Lindb. Relaciones con otras especies. Cryptogamie, Bryol.Lichénol., 9(4):343-352, 4 pl. fig., 1 carte.

GUERRA, J., J.A. GIL \& J. VARO -1981 - Aportación al conocimiento de las clases PogonatoDicranelletea heteromallae Hübschmann 1975 y Ceratodo-Polytrichetea piliferi Hübschmann 1967 en el sur de España y Portugal. Lazaroa, 3:101-119, 1 fig. 5 tabl.

HÉBRARD, J.P. -1979- Complément à l'étude de la bryoflore du parc national de Port-Cros et notes sur le pH édaphique. Trav. Scient. Parc Nation. Port-Cros, 5:35-58, 10 tabl., 4 fig.

HÉBRARD, J.P. -1980- Contribution à l'étude de la végétation muscinale de quelques formations du maquis corse: les pelouses sèches ou humides sur silice. Bull. Soc. Linn Provence, 32(1979):15-45, 7 tabl.

HÉBRARD, J.P. -1981- Contribution à l'étude de la végétation bryophytique des forêts de Quercus ilex, de Quercus suber et des maquis bas à Rosmarinus officinalis dans le Cap Corse. Lejeunia, N.S., 106:1-20, 6 tabl.

HÉBRARD, J.P. -1982a- Mousses et hépatiques du bassin méditerranéen. Beih.Nova Hedwigia, 71:369-386. 
HÉBRARD, J.P. - 1982b- Remarques sur l'écologie de quelques mousses peu connues dans les Bouches-du-Rhône et la partie occidentale et méridionale du département du Var. Bull. Soc. Linn. Provence, 33(1981):25-49, 4 tabl., 2 fig.

HÉBRARD, J.P. -1984a- Remarques sur la répartition géographique et l'écologie d'Anacolia webbii (Mont.) Schimp., d'Entosthodon curvisetus (Schwaegr.) C. Müll. et de Grimmia tergestina Tomm. ex B., S. et G. var. tergestina en Corse. Cryptogamie, Bryol. Lichénol., 5(1-2):33-45, 6 tabl., 2 fig.

HÉBRARD, J.P. -1984b- Contribution à l'étude du peuplement bryophytique des pinèdes de pin d'Alep sur substrat calcaire dans les Bouches-du-Rhône et le Var sud-occidental. Ecol. Medit., 10(1-2):183-203, 3 tabl., 4 fig.

HÉBRARD, J.P. -1986- Note de bryologie corse: muscinées rares, méconnues ou nouvelles pour l'île. Bull. Soc. Bot. Centre-Ouest, N.S., 17:151-167.

HÉBRARD, J.P. -1987a- Aperçu sur la bryoflore des terrains calcaires, aux confins septentrionaux des département des Bouches-du-Rhône et du Var.Bull. Soc.Linn. Provence, 38(1986): 127-138.

HÉBRARD, J.P. -1987b- Etude comparée du peuplement bryophytique des chênaies pubescentes de «bas-fond» et de «plateau» en forêt domaniale de la Gardiole de Rians (Var, France). Cryptogamie, Bryol. Lichénol., 8(2):109-146, 13 tabl., 7 fig.

HÉBRARD, J.P. -1987- Etude comparée de la végétation bryophytique des parties basses et moyennes des troncs de chêne vert et de chêne pubescent (peuplements jeunes) dans la forêt domaniale de la Gardiole de Rians (Var, France). Bull. Soc. Bot. Centre-Ouest N.S., 18:125-144, 6 tabl., 7 fig.

HÉBRARD, J.P. -1988- Contribution à l'étude des bryophytes de la réserve naturelle de la presqu'île de Scandola (Corse). Candollea, 43:189-197, 1 tabl., 3 fig.

HÉBRARD, J.P. \& C. ROLANDO -1985- Etude comparée du peuplement bryophytique de taillis de chêne vert d'âge différent en forêt domaniale de la Gardiole de Rians (Var, France). Ecol. Medit., 11(2-3):87-110, 14 tabl., 6 fig.

HERRNSTADT, I., C.C. HEYN \& M.R. CROSBY -1980- New data on the moss genus Gigaspermum. Bryologist., 83(4):536-541, 12 fig.

HERRNSTADT, I., C.C. HEYN, R. BEN-SASSON \& M.R. CROSBY -1982- New records of mosses from Israel. Bryologist, 85(2):214-217.

JOVET-AST, S. -1986-Les Riccia de la région méditerranéenne. Cryptogamie,Bryol.Lichénol., 7(3) (supplément):287-431.

KRAMER, W. -1980- Tortula Hedw. sect. Rurales De Not. (Pottiaceae, Musci) in der östlichen Holarktis. Bryophyt. Bibliotheca, 21:1-224.

LECOINTE, A. -1979- Intérêts phytogéographiques de la bryoflore normande: 1 - Les cortèges cosmopolite et méditerranéen S.L. Bull. Soc. Linn. Normandie, 107:61-70, 1 fig.

LECOINTE, A. -1981a- Intérêts phytogéographiques de la bryoflore normande: 2 - Le cortège atlantique S.L. Bull. Soc. Linn. Normandie, 108:51-60, 1 tabl.

LECOINTE, A. -1981b- Intérêts phytogéographiques de la bryoflore normande: 3 - Le cortège circumboréal S.L. Bull. Soc. Linn. Normandie, 109:55-66, 1 tabl.

LECOINTE, A. -1982- Etat actuel des travaux sur la floristique et la chorologie des bryophytes en France. Lejeunia N.S., 107:23-25.

LECOINTE, A. -1988- Intérêts phytogéographiques de la bryoflore normande: 4 - Additions, corrections, spectres biogéographiques et écologiques. Bull. Soc. Linn. Normandie, 110111:23-40, 2 tabl., 3 pl. fig.

LLORET, R. -1987- Bryoerythrophyllum inaequalifolium (Tayl.) Zander, new to the european 
continent. Lindbergia, 13(3):127-129, 1 fig.

LO GIUDICE R. \& M. PRIVITERA -1987a- Sulla briovegetazione del Vallone Murapane (Piazza Armerina). Boll. Acc. Gioenia Sci. Nat., 20(330):149-163, 6 tabl.

LO GIUDICE, R. \& M. PRIVITERA -1987b- Sulla florula e vegetazione briofitica della grotta Garrone (Mte Pizzuta) Piana degli Albanesi. Boll. Acc. Gioenia Sci. Nat., 20(330):165170,1 tabl.

MARTINCIC, A. -1980- Beiträge zur Kenntnis der Moosflora Jugoslawiens, II. Sar Planina. Biol. Vest., 28(2):87-102.

OCHYRA, R., C. SERGIO \& R. SCHUMACKER -1988- Racomitrium lamprocarpum (C. Müll.) Jaeg. an austral moss disjunct in Portugal, with taxonomic and phytogeographic notes. Bull. Jard. Bot. Nat. Belg., 58:225-258, 7 fig.

OZENDA, P. -1975- Sur les étages de végétation dans les montagnes du bassin méditerranéen. Doc. Cartogr. Ecol., 16:1-32, 19 fig.

OZENDA, P. -1982- Les végétaux dans la biosphère. Paris: Doin, 431 p.

OZENDA, P. \& H. WAGNER -1975- Les séries de végétation de la chaîne alpine et leurs équivalences dans les autres systèmes phytogéographiques. Doc. Cartogr. Ecol., 16:4964, 1 fig.

PAVLETIC, Z. \& V. PULEVIC -1980- A contribution to the bryophytic flora of Montenegro. The montene grin academy of sciences and arts, glasnik of the section of natural sciences, 3:111-131, 2 tabl.

PIERROT, R.B. -1986- Crossidium aberrans Holz. et Bartr., mousse nouvelle pour la France. Bull. Soc. Bot. Centre Ouest N.S., 17:149-150.

PIERROT, R.B., M.A. ROGEON \& J.M. HOUMEAU -1983- Liste des bryophytes observées pendant la 9e session extraordinaire de la S.B.C.O.: «Grands causses cévenols et LévezouAigoual». Bull. Soc. Bot. Centre Ouest N.S., 14:141-147.

PIERROT, R.B. \& P. BOUDIER, E. DEBON, P. PLAT, J. SAPALY, R. SORNICLE \& A. TERRISSE -1987- L'année bryologique 1986. Bull. Soc. Bot. Centre Ouest N.S., 18:117120.

PRESTON, C.D. -1981 - A check-list of greek liverworts. Journ. of Bryology, 11(3):537-553.

PRESTON, C.D. -1984a- A check-list of greek mosses. Journ. of Bryology, 13(1):43-95.

PRESTON C.D. -1984b- A check-list of greek liverworts: addendum. Journ. of Bryology, 13(1):97-100.

PRIVITERA, M. \& R. LO GIUDICE -1981- Vegetazione muscinale della faggeta del bosco di Malabotta (Peloritani). Cryptogamie, Bryol. Lichénol., 2(2):181-200, 7 tabl., 4 fig.

PRIVITERA, M. \& R. LO GIUDICE -1985- Contributo allo studio della vegetazione muscinale del territorio di Santo Pietro (Sicilia meridionale): sugherete, leccete, cisteti. Ecol. Medit., 11(4):75-86, 7 tabl., 2 fig.

PRIVITERA, M. \& R. LO GIUDICE-1986-Sulla briovegetazione dei tufi calcarei dell'Ennese (Sicilia). Cryptogamie, Bryol. Lichénol., 7(2):129-139, 3 tabl., 1 fig.

PRIVITERA, M. \& R. LO GIUDICE -1988- Sulla presenza di Bryum dunense Smith et Whitehouse in Sicilia. Giorn. Bot. Ital., 122, suppl. 1:39.

PUCHE, F., A. CASAÑ \& J.J. HERRERO-BORGOÑON -1987- El género Exormotheca Mitt. (Hepaticophytina) en la Península Ibérica. Acta Bot. Malacit., 12:246-248, 1 fig.

RAIMONDO, F.M. \& M.G. DIA -1979- Note biogeografiche II. Cryphaea heteromalla (Hedw.) Mohr in Sicilia. Atti Accad. Sci. Lett. Arti di Palermo ser. 4(38, 1) (1978-1979):318, 1 tabl., 1 fig., 3 phot.

RAIMONDO F.M., M.G. DIA \& C. CORTINI PEDROTTI -1988- Stato dell'esplorazione 
briogeografica en Sicilia. Giorn. Bot. Ital., 122, suppl. 1: 220.

RAIMONDO, F.M., M.G. DIA \& M. ROSSITO -1981- L'Eucladio-Adiantetum Br.-B1. 1931 in Sicilia. Atti Accad. Sci. Lett. Arti di Palermo, 40, ser. 4:1-12, 1 tabl., 2 fig.

RAIMONDO, F.M., A. SCIALABBA \& M.G. DIA -1980- Note briogeografiche III. Distribuzione in Italia di Aulacomnium palustre (Hedw.) Schwaegr. ed ecologia della specie nelle stazioni siciliane (Bryales, Aulacomniaceae). Naturalista Sicil., 4(4) (3-4):7999, 2 tabl., 6 fig.

ROS, R.M. \& J. GUERRA - 1985- Aportación al conocimiento de la brioflora del sureste de España. An. Jard. Bot. Madrid, 41(2):257-266, 6 fig.

ROS, R.M. \& J. GUERRA -1986- Crossidium aberrans Holz. et Bartr. (Musci) novedad para la flora europea. Cryptogamie, Bryol. Lichénol., 7(1):71-75, 1 fig., 1 carte.

ROS, R.M.\& J. GUERRA - 1987a- Catálogo de briófitos terrícolas de la región de Murcia (SE de España). Candollea, 42:577-599, 1 fig.

ROS, R.M. \& J. GUERRA -1987b- Vegetación briofítica terrícola de la región de Murcia (sureste de España). Phytocoenologia, 15(4):505-567, 14 tabl., 9 fig.

ROS ESPIN, R.M. -1987- Riella cossoniana Trab., nueva hepática para la flora europea. Cryptogamie, Bryol. Lichénol., 8(3):227-233, 1 pl. phot., 1 fig., 1 carte.

ROSSELLO, J.A. -1981- Notes sobre la brioflora balear. 1. Briófites noves per Balears. Boll. Soc. Hist. Nat. Balears, 25:39-51, 6 fig.

ROSSELLO, J.A. -1985- Notes sobre la brioflora balear, III. Collect. Bot., 16:63-66.

ROSELLO, J.A. - 1986- Notes sobre la brioflora balear, IV. Acta Bot. Malacit., 11:77-82.

ROSSELLO, J.A. - 1987- Notas sobre la brioflora balear, 5. Acta Bot. Malacit., 12:81-85, 2 fig.

SCHUMACKER, R., I. BISANG \& C. CORTINI PEDROTTI -1988- Amblystegium compactum (C. Muell.) Aust. (Musci) in Italy. Giorn. Bot. Ital., 122:25-29, 1 fig.

SERGIO, C. -1980- Sur la présence de Mielichhoferia pontevedrensis Luis. au Portugal. Cruptogamie, Bryol. Lichénol., 1(1):91-94, 2 fig.

SERGIO, C. - 1982a-L'état des prospections bryologiques au Portugal. Lejeunia N.S., 107:4547.

SERGIO, C. -1982b- Contribuição para o conhecimento do genero Ephemerum Hampe na Península Ibérica. Acta Bot. Malacit., 7:87-96, 5 fig.

SERGIO, C. - 1984a- The distribution and origin of macaronesian bryophyte flora. Journ. Hattori Bot. Lab., 56:7-13, 2 tabl., 2 fig.

SERGIO, C. -1984b- Estudo taxonómico, ecológico e corológico de Gymnostomum luisieri (Sergio) Sergio ex Crundw. na Península Ibérica. An. de Biologia 2 (sección especial, 2):357366, 3 tabl., 2 fig.

SERGIO, C. -1985a- Duas novas especies de Frullania para a flora briológica de Portugal. In Sergio C. et al.: notulae bryoflorae lusitanicae I. Portug. Acta Biol. (B), 14:181-184, 1 fig.

SERGIO, C. - 1985b- Notas acerca do genero Fossombronia Raddi em Portugal. In Sergio C. et al.: notulae bryoflorae lusitanicae I. Portug. Acta Biol. (B), 14:188-194, 2 fig.

SERGIO, C. -1987-Contribuição para o estudo taxonómico e fitogeográfico de Anthoceros caucasicus Steph. na Península Ibérica e Macaronesia. Act. VI Simp. Nac. Bot. Cript.: 605613, 3 fig.

SERGIO, C. -1988- Morphological, karyological and phytogeographic observations on Entosthodon curvisetus (Schwaegr.) C Müll. as a basis for a new genus, Funariella Sergio (Funariaceae: Musci). Orsis, 3:5-13, 4 fig.

SERGIO, C. \& J.P. HÉBRARD -1982- Orthothecium duriaei (Mont.) Besch. Etude systématique, écologique et phytogéographique. Collect. Bot., 13(1) IV simposi de botanica 
criptogamica. Barcelona 1982:247-255, 2 fig.

SERGIO, C. \& M. SIM-SIM -1985- a vegetação briológica das formações calcárias de Portugal III. Portug. Acta Biol. (B), 14:105-154, 2 tabl., 5 fig.

SERGIO, C., C. CASAS, R.M. CROS, M. BRUGUES \& M. SIM-SIM -1987- Bryophyte vegetation and ecology of calcareous areas in the Iberian Peninsula. Symposia Biologica Hungarica, 35:423-446, 1 tabl.

SERGIO, C., M. SIM-SIM, C. CASAS, M. BRUGUES \& R.M. CROS -1987- Notulae bryoflorae lusitanicae II. Alguns musgos novos ou raros para a flora de Portugal, recentemente encontrados no maciço calcário estremenho. Ref. Biol. u Aveiro, 1:47-52.

SERGIO, C., M. SIM-SIM, C. CASAS, M. BRUGUES \& R.M. CROS -1988- A vegetação briológica das formações calcárias de Portugal IV. O maciço calcário estremenho - Serras de Aire, Candeeiros e Sicó. Mem. Soc. Brot, 28:93-135, 1 tabl., 6 fig.

SERGIO, C., M. SIM-SIM, C. CASAS, R.M. CROS \& M. BRUGUES -1984- A vegetação briológica das formações calcárias de Portugal II. O Barrocal albarfio e o promontorio sacro. Bol. Soc. Brot. ser. 2, 57:275-307, 1 tabl., 4 fig.

SLACK, N.G. -1977- Species diversity and community structure in Bryophytes: New York state studies. New York State Museum Bull., $428: 76$ p., 9 tabl., 30 fig., 40 phot.

TOWNSEND, C.C. -1986- Records of greek mosses. Journ. of Bryology, 14(1):135-137.

WERNER, J. -1989- Observations bryologiques en Provence - Côte d'Azur. Bull. Soc. Linn. Provence, 40:69-72.

WERNER, J. \& J.P. HÉBRARD -1986- Tortula caninervis (Mitt.) Broth. subsp. spuria (Amann) W. Kramer var. spuria (Pottiaceae, Musci) à Touët-sur-Var (Alpes Maritimes), nouveau pour la bryoflore de France. Cryptogamie, Bryol.Lichénol., 7(4):487-493, 1 fig.

WHITEHOUSE, H.L.K. -1984- Survival of a moss, probably Dicranella staphylina, in soil stored for nearly 50 years. Journ. of Bryology, 13(1):131-133.

(1) Abréviations des pays comme dans Flora Europaea

(2) Localité inédite: Rif (Maroc)

(Melilla, Septiembre de 1989)

Dirección del autor: Chargé de recherche au C.N.R.S., Institut méditerranéen d'écologie et de paléoécologie, laboratoire de botanique et d'écologie méditerranéenne. Faculté des Sciences et Techniques de Saint-Jêrome. Avenue de l'Escadrille Normandie-Niemen. F 13397 Marseille Cedex 13. 